\title{
Genetic diversity of mitochondrial DNA from Litopenaeus vannamei broodstock used in northwestern Mexico
}

\section{Diversidad genética del ADN mitocondrial en reproductores de Litopenaeus vannamei utilizados en el noroeste de México}

\author{
Fernando Mendoza-Cano ${ }^{1,2}$, José Manuel Grijalva-Chon ${ }^{1 *}$, Ricardo Pérez-Enríquez ${ }^{3}$, \\ Josefina Ramos-Paredes ${ }^{1,4}$, Alejandro Varela-Romero ${ }^{1}$ \\ ${ }^{1}$ Departamento de Investigaciones Científicas y Tecnológicas, Universidad de Sonora, Av. Colosio s/n, \\ entre Sahuaripa y Reforma, Hermosillo 83000, Sonora, México. \\ ${ }^{2}$ Laboratorio de Referencia, Análisis y Diagnóstico en Sanidad Acuícola, Centro de Investigaciones Biológicas \\ del Noroeste, Calle Hermosa 101, Col. Los Ángeles, Hermosillo 83106, Sonora, México (permanent address). \\ ${ }^{3}$ Centro de Investigaciones Biológicas del Noroeste, Instituto Politécnico Nacional 195, Col. Playa Palo de \\ Santa Rita Sur, La Paz 23096, Baja California Sur, México. \\ ${ }^{4}$ Laboratorio Especializado de Biología Molecular, Servicio Nacional de Sanidad, Calle Esteban Sarmiento 35, \\ Col. Matanza, Hermosillo 83080, Sonora, México (current address).
}

* Corresponding author. E-mail: mgrijal@guayacan.uson.mx

\begin{abstract}
Shrimp cultivation in Mexico is based on the whiteleg shrimp, Litopenaeus vannamei, with a production of about $100,320 \mathrm{t}$ in 2012. Postlarvae are produced in hatcheries, where the selection process is geared towards producing lineages with better productive parameters and resistance to some diseases; however, the crossing of related organisms may reduce genetic variability, resulting in inbreeding depression. In this study we analyzed the sequences of the mitochondrial DNA control region of 425 shrimp from five hatcheries and of 29 wild whiteleg shrimp. The results suggest the presence of two dominant haplotypes in a monophyletic group of organisms used as broodstock and in wild whiteleg shrimp; this finding suggests a common origin. Low levels of genetic variability in some hatcheries highlight the importance of monitoring genetic diversity and supervising breeding programs to prevent loss of haplotypes.
\end{abstract}

Key words: broodstock, genetic diversity, mitochondrial DNA, Litopenaeus vannamei, sequence analysis.

RESUMEN. El cultivo de camarón en México se basa en el camarón blanco, Litopenaeus vannamei, con una producción de aproximadamente 100,320 t en 2012. El suministro de postlarvas proviene de laboratorios de producción cuyo proceso de selección está dirigido a producir linajes con parámetros más productivos y resistencia a ciertas enfermedades; sin embargo, la cruza de organismos emparentados puede reducir la variabilidad genética, dando como resultado una depresión endogámica. En este estudio se analizaron las secuencias de la región control del ADN mitocondrial de 425 camarones procedentes de cinco laboratorios de producción de postlarvas y 29 camarones silvestres. Los resultados evidencian la presencia de dos haplotipos dominantes en un grupo monofilético de los organismos utilizados como reproductores y en los organismos silvestres; este hallazgo sugiere la hipótesis de un origen común. Debido a los bajos valores de variabilidad genética en algunos laboratorios, se resalta la importancia de vigilar la diversidad genética y supervisar los programas de reproducción para evitar la pérdida continua de haplotipos.

Palabras clave: reproductores, diversidad genética, ADN mitocondrial, Litopenaeus vannamei, análisis de secuencias.

\section{INTRODUCTION}

Penaeid shrimp cultivation contributes $9 \%$ of the world's aquaculture production, with a mean annual growth of $18 \%$ since the 1970s (Benzie 2009). Its expansion has generated a large demand for hatchery postlarvae produced under controlled conditions. Usually, broodstock selection is based on phenotypic traits to obtain lineages with better production parameters and disease resistance. Artificial selection usually increases mating of closely related organisms, leading to reduced genetic variability and ability to produce viable offspring. Inbreeding can affect survival, growth, and reproductive quality (Sbordoni et al. 1986, 1987; De Donato et al.

\section{INTRODUCCIÓN}

El cultivo de camarones peneidos contribuye con el $9 \%$ de la producción acuícola mundial, con un crecimiento medio anual del 18\% desde 1970 (Benzie 2009). Su expansión ha generado una gran demanda de postlarvas producidas en laboratorio bajo condiciones controladas. Generalmente, el pie de cría se selecciona con base en sus rasgos fenotípicos para obtener linajes con mejores parámetros productivos y con resistencia a enfermedades. El proceso de selección artificial generalmente incrementa la cruza de organismos estrechamente relacionados, lo cual reduce la variabilidad genética y la capacidad de dejar descendencia viable. La 
2005; Moss et al. 2007). Surveillance of the genetic structure in hatchery facilities is thus very important (Álvarez-Jurado 1987, Benzie and Williams 1996). Breeding programs and management of fishery resources require detailed information of the genetic structure of shrimp populations and its associated diversity (Chu et al. 2003). Benzie (2009) reviewed the most relevant information on genetic resources of penaeid shrimp.

In Mexico, production of cultivated shrimp, mainly Litopenaeus vannamei, during 2012 was 100,320 t, exceeding wild shrimp catches (CONAPESCA 2012). Shrimp farming is mainly concentrated along the coast of the Gulf of California. In this region, commercial hatcheries produce up to 10 billion postlarvae each year to supply shrimp farmers. Mexican producers of shrimp larvae need to understand the genetic variability of their broodstock. Several genetic markers have been used, including allozymes (Ramos-Paredes and Grijalva-Chon 2003, Soto-Hernández and Grijalva-Chon 2004, Rivera-García and Grijalva-Chon 2006) and random amplified polymorphic DNA (RAPD) (Valerio-García and Grijalva-Chon 2008). Perez-Enriquez et al. (2009) analyzed the genetic diversity of broodstock from six commercial hatcheries using microsatellites, and the results showed moderate diversity, major inbreeding, and evidence of a common origin of the broodstock. This study aims to complement that of Perez-Enriquez et al. (2009) by using sequence analysis of the mitochondrial DNA control region of broodstock from five hatcheries to estimate genetic variability and strengthen the hypothesis of a common origin.

\section{MATERIALS AND METHODS}

\section{Amplifying and sequencing of the mitochondrial DNA control region}

Broodstock of 425 shrimp from five hatcheries (A, B, C, $\mathrm{E}$, and F) located in northwestern Mexico were analyzed. Details of their origin and DNA isolation procedures are given in Perez-Enriquez et al. (2009). Additionally, 29 wild whiteleg shrimp reported by Valerio-García and GrijalvaChon (2008) were analyzed.

DNA primers RV1 (5'-TATAACCGCGGCTGCTGGCAC$3^{\prime}$ ) and FW1 (5'-GGTATGAGCCCACGAGCTTGC-3') were designed from the NC_009626 sequence in GenBank to amplify a $1452 \mathrm{bp}$ segment. The segment partly includes a segment of the $12 \mathrm{~S}$ rRNA gene, the entire mitochondrial control region, and a portion of the tRNA ${ }^{\text {met }}$ gene. This region extends from base number 14,766 to base number 228 of the NC_009626 sequence. Polymerase chain reactions (PCR) were performed using PuRe Taq Ready-to-Go PCR beads (GE Healthcare Life Sciences, Uppsala, Sweden) with $1 \mu \mathrm{L}$ of each primer, $2 \mu \mathrm{L}$ DNA extract, and $21 \mu \mathrm{L}$ PCR grade water. PCR conditions were an initial denaturation step at $95^{\circ} \mathrm{C}$ for $5 \mathrm{~min}, 35$ cycles of $95^{\circ} \mathrm{C}$ for $50 \mathrm{~s}, 60^{\circ} \mathrm{C}$ for $50 \mathrm{~s}$, $72{ }^{\circ} \mathrm{C}$ for $90 \mathrm{~s}$, and a final extension step at $72{ }^{\circ} \mathrm{C}$ for $7 \mathrm{~min}$. The PCR products were visualized on $2 \%$ agarose gel endogamia puede afectar la supervivencia, el crecimiento y la calidad reproductiva (Sbordoni et al. 1986, 1987; De Donato et al. 2005; Moss et al. 2007). Es por ello que es muy importante conocer y vigilar la estructura genética de los reproductores utilizados en los laboratorios de producción (ÁlvarezJurado 1987, Benzie y Williams 1996). Los programas de mejoramiento genético y el manejo de los recursos pesqueros necesitan de información detallada de la estructura genética de las poblaciones de camarón y de su diversidad asociada (Chu et al. 2003). Benzie (2009) hizo una revisión de la información más relevante sobre los recursos genéticos de los camarones peneidos.

En México, la producción de camarón de cultivo, principalmente Litopenaeus vannamei, en 2012 fue de 100,320 t, excediendo la producción de captura por pesca (CONAPESCA 2012). El cultivo de camarón se concentra principalmente en las costas del golfo de California. En esta región, los laboratorios de producción de postlarvas producen hasta 10 mil millones de organismos anualmente para satisfacer las necesidades de las granjas camaroneras. Los productores mexicanos de postlarvas de camarón necesitan conocer la variabilidad genética en los organismos empleados como pie de cría (reproductores); para este propósito, se han utilizado varios marcadores genéticos, incluyendo alozimas (Ramos-Paredes y Grijalva-Chon 2003, Soto-Hernández y Grijalva-Chon 2004, Rivera-García y Grijalva-Chon 2006) y ADN polimórfico al azar (Valerio-García y Grijalva-Chon 2008). Perez-Enriquez et al. (2009) analizaron la diversidad genética en reproductores de seis laboratorios de producción mediante microsatélites, y los resultados indicaron diversidad moderada, alta endogamia y evidencia de un origen común del pie de cría. El objetivo del presente trabajo es complementar el estudio de Perez-Enriquez et al. (2009) mediante un análisis de secuencias de la región control del ADN mitocondrial del pie de cría de cinco laboratorios de producción para estimar la variabilidad genética y fortalecer la hipótesis de un origen común.

\section{MATERIALES Y MÉTODOS}

\section{Amplificación y secuenciación de la región control del ADN mitocondrial}

Se analizaron 425 organismos reproductores de $L$. vannamei de cinco laboratorios de producción de postlarvas (A, B, C, E y F) localizados en el noroeste de México. Los detalles de su origen y de los procedimientos de extracción de ADN están documentados en Perez-Enriquez et al. (2009). Además, se incluyó una muestra de 29 organismos silvestres que corresponde a la registrada por Valerio-García y Grijalva-Chon (2008).

Los oligos RV1 (5'-TATAACCGCGGCTGCTGGCAC3') y FW1 (5'-GGTATGAGCCCACGAGCTTGC-3') fueron diseñados a partir de la secuencia NC_009626 del GenBank para amplificar un producto de 1452 pares de bases (pb). 
(Sigma-Aldrich) prepared with TAE buffer $1 \times$ and stained with ethidium bromide (Sigma-Aldrich). A molecular weight marker of $100 \mathrm{bp}$ (Invitrogen10488-058) was used to estimate the size of the obtained products. PCR products were sent to Macrogen in Seoul (Korea) for purification and sequencing in both directions. The sequences were assembled, corrected for ambiguities, and adjusted only to the mitochondrial control region, using ChromasPro v1.41 software (Technelysium, Brisbane, Australia).

\section{Broodstock genetic diversity by sequence analysis}

The analysis considered each base position as a locus to record polymorphic sites. Similar to Perez-Enriquez et al. (2009), all individuals within a hatchery were pooled to represent the hatchery's genetic composition and gene diversity. Haplotypes in all samples were identified and their frequencies calculated. Data analysis was performed with Arlequin v3.5 software (Excoffier et al. 2005). Gene and nucleotide diversities were calculated with the same software, and an analysis of molecular variance (AMOVA) was performed. To determine the degree of difference between hatcheries, the $F_{S T}$ value between paired samples was calculated, and the exact test, analogous to Fisher's test, was performed using 100,000 Markov-chain steps and 10,000 dememorization steps.

\section{Origin of broodstock in Mexico}

To determine the origin of $L$. vannamei broodstock in Mexico and the phylogenetic relationship with wild shrimp populations, PAUP* 4.0b software (Swofford 2002) was used with the complete mitochondrial control region sequences for each haplotype in our samples and those reported in GenBank for this species and other species of the genus.

All sequences were aligned using Clustal X v2.1 (Larkin et al. 2007). For the construction of the phylogenetic relationship between the populations and sequences deposited in GenBank of the same species and other genera of the family, maximum parsimony and maximum likelihood methods were used. We used complete nucleotide sequences for both approaches to obtain the most parsimonious trees that showed the groups or clades within each species, using tree bisection-reconnection (TBR) as the branch swapping search with PAUP*. All characters were equally weighted. Insertions and deletions (indels) were treated as missing and starting trees were obtained by 1000 random stepwise additions. Nodal support was estimated by nonparametric bootstrap (1000 pseudo-replicates, 10 random additions) proportions (Felsenstein 1985) and decay indices (Bremer 1994) using PAUP*. The consensus tree was constructed using PAUP*. We calculated the hierarchical likelihood rate (HLRT; Posada and Buckley 2004) with ModelTest v3.06 (Posada and Crandall 1998) to estimate the optimal model of nucleotide substitution in our maximum likelihood analysis. With this model, we conducted a maximum likelihood search
Este producto incluye parcialmente un segmento del gen ARNr 12S, toda la región control y una porción del gen ARN de transferencia de metionina (ARNt ${ }^{\text {met }}$ ). Esta región comprende desde la base 14,766 a la 228 de la secuencia NC_009626. Las reacciones en cadena de la polimerasa (PCR, por sus siglas en inglés) se llevaron a cabo con esferas liofilizadas PuRe Taq Ready-to-Go (GE Healthcare Life Sciences, Uppsala, Sweden); cada esfera se disolvió en un volumen de $21 \mu \mathrm{L}$ de agua grado PCR, y se le agregaron $2 \mu \mathrm{L}$ de los oligos FW1 y RV1, y $2 \mu \mathrm{L}$ del ADN a analizar. Las condiciones de amplificación fueron desnaturalización inicial a $95^{\circ} \mathrm{C}$ durante $5 \mathrm{~min}, 35$ ciclos de $95^{\circ} \mathrm{C}$ durante $50 \mathrm{~s}$, $60{ }^{\circ} \mathrm{C}$ durante $50 \mathrm{~s}, 72{ }^{\circ} \mathrm{C}$ durante $90 \mathrm{~s}$ y un paso final a $72{ }^{\circ} \mathrm{C}$ durante $7 \mathrm{~min}$. Los productos de PCR se visualizaron mediante geles de agarosa (Sigma-Aldrich) preparados al 2\% con amortiguador TAE $\times 1$ y teñidos con bromuro de etidio (Sigma-Aldrich). Se utilizó un marcador de peso molecular de $100 \mathrm{pb}$ (Invitrogen 10488-058) para estimar el tamaño del producto obtenido. Los productos de PCR fueron enviados a Macrogen (Seúl, Corea) para su purificación y secuenciación en ambos sentidos. Las secuencias obtenidas fueron ensambladas, corregidas de ambigüedades y ajustadas solamente a la región control mitocondrial con el programa ChromasPro v1.41 (Technilysium, Brisbane, Australia).

\section{Diversidad genética en reproductores mediante el análisis de secuencias}

El análisis consideró a cada posición de bases como un locus para registrar los sitios polimórficos. Al igual que Perez-Enriquez et al. (2009), todos los individuos de un laboratorio se combinaron para representar la composición y diversidad genética del laboratorio. Se identificaron a los haplotipos y se calcularon sus frecuencias en todas las muestras. El análisis de datos se realizó con el programa Arlequin v3.5 (Excoffier et al. 2005). Con el mismo programa se calcularon las diversidades de genes y nucleotídicas, y se realizó un análisis de varianza molecular (AMOVA). Para determinar el grado de diferenciación entre los laboratorios, se calculó el valor de Fst entre muestras pareadas y se realizó la prueba exacta, análoga a la de Fisher, a través de la cadena de Markov con 100,000 pasos y 10,000 pasos de desmemorización.

\section{Origen del pie de cría en México}

Para determinar el origen del pie de cría de L. vannamei en México y la relación filogenética con poblaciones silvestres, se utilizó el programa PAUP* 4.0b (Swofford 2002) con las secuencias completas de la región control de cada uno de los haplotipos de nuestras muestras y de las registradas en el GenBank para esta especie y otras especies del género.

Todas las secuencias fueron alineadas con Clustal X v2.1 (Larkin et al. 2007). Para la construcción de la hipótesis de relación filogenética entre las poblaciones de estudio y otras 
with a branch-swapping algorithm to find the tree topology and greater likelihood values using PAUP*. This tree was used as the initial topology for calculating the nonparametric re-sampling of the data (1000 pseudo-replicates) to estimate the support of the nodes in the maximum likelihood tree. The cladogram was constructed using the FigTree v1.4 software (http://tree.bio.ed.ac.uk/software/figtree/).

\section{RESUlts}

Thirty-six haplotypes were obtained from the sequence analysis of the samples from five hatcheries and they were registered in GenBank (accession numbers GQ857079 to GQ857122). All sequences were compared with the mitochondrial control region of NC_009626 and comparisons provided $94-99 \%$ homology. The length of the sequences fluctuated between 996 and 998 nucleotides because of indels; polymorphic sites ranged between 78 and 114 . The wild sample showed the lowest number of polymorphic sites (table 1).

Two haplotypes ( $\mathrm{H} 1$ and $\mathrm{H} 2)$ showed high frequency in all samples, with $76 \%$ in those from hatchery $\mathrm{B}$ and up to $96 \%$ in those from hatchery $\mathrm{C}$ (table 2). Other haplotypes had very low frequencies and, with the exception of haplotype 29, were distinct to individual hatcheries. Hatchery E samples and the wild sample had the fewest haplotypes (5), and hatchery A samples had the most (15). Hatchery B samples had the highest diversity and hatchery $\mathrm{C}$ samples the lowest. Diversity of the wild sample was lower than in the samples from three hatcheries (table 1).

Genetic differentiation analysis, based on the AMOVA, showed that $86 \%$ of molecular variance occurs within hatcheries; global $F_{S T}$ was 0.14 , which was significantly different from zero $(P<0.001)$. Of the 15 paired comparisons, the $F_{S T}$ analysis showed that $66 \%$ were significant, agreeing in general with the exact test. Both tests showed that the wild sample was not different from hatcheries A, E, and F (table 3).

We generated 1109 nucleotide sites in the alignment due to indels, of which 607 were parsimoniously informative, secuencias depositadas en el GenBank de la misma especie y de otros géneros de la familia, se utilizaron los métodos de máxima parsimonia y máxima verosimilitud. Se utilizaron las secuencias nucleotídicas completas para ambas aproximaciones para obtener los árboles más parsimoniosos que mostraran los grupos o clados en cada especie analizada, utilizando bisección-reconexión de árboles (TBR) como búsqueda de intercambio de ramas con PAUP*. Todos los caracteres tuvieron el mismo peso. Las inserciones y eliminaciones (indels) fueron tratadas como faltantes, y los primeros árboles se obtuvieron por 1000 adiciones por pasos inteligentes al azar. El sostenimiento de los nodos se estimó con proporciones de remuestreo no paramétrico (1000 pseudoréplicas, 10 adiciones al azar) (Felsenstein 1985) y con el índice de decaimiento (Bremer 1994) utilizando PAUP*. EL árbol consenso fue construido utilizando PAUP*. Se calculó la tasa jerárquica de verosimilitud (HLRT) (Posada y Buckley 2004) con ModelTest v3.06 (Posada y Crandall 1998) para estimar el modelo óptimo de sustitución nucleotídica en el análisis de máxima verosimilitud. Con éste modelo se realizó una búsqueda de máxima verosimilitud mediante el algoritmo de intercambio de ramas para encontrar la topología del árbol y los valores de mayor verosimilitud utilizando PAUP*. Este árbol fue utilizado como topología inicial para el cálculo del remuestreo no paramétrico de los datos (1000 pseudoréplicas) para estimar el sostenimiento de los nodos en el árbol de máxima verosimilitud. El cladograma fue construido con el programa FigTree v1.4 (http://tree.bio.ed.ac.uk/software/figtree/).

\section{RESUltados}

Se obtuvieron 36 haplotipos del análisis de secuencias de las muestras de los cinco laboratorios y fueron registrados en el GenBank (números de acceso GQ857079 a GQ857122). Todas las secuencias se compararon con la región control mitocondrial de la secuencia NC_009626, y las comparaciones mostraron una homología del 94-99\%. La longitud de las secuencias fluctuaron entre 996 y 998 nucleótidos debido a las indels, y los sitios polimórficos variaron entre 78 y 114 .

Table 1. Basic properties of sequences and molecular diversity indices of the mitochondrial DNA control region in Litopenaeus vannamei broodstock from five hatcheries (A, B, C, E, F) and a wild sample from northwestern Mexico. Mean \pm standard deviation.

Tabla 1. Propiedades básicas e índices de diversidad molecular de la región control del ADN mitocondrial en reproductores de Litopenaeus vannamei de cinco laboratorios (A, B, C, E, F) y de una muestra silvestre del noroeste de México. Promedio \pm desviación estándar.

\begin{tabular}{lccccccc}
\hline Sample & $\begin{array}{c}\text { No. of } \\
\text { gene copies }\end{array}$ & $\begin{array}{c}\text { No. of } \\
\text { loci }\end{array}$ & $\begin{array}{c}\text { No. of } \\
\text { usable loci* }\end{array}$ & $\begin{array}{c}\text { No. of } \\
\text { polymorphic sites }\end{array}$ & $\begin{array}{c}\text { Gene } \\
\text { diversity }\end{array}$ & $\begin{array}{c}\text { Nucleotide } \\
\text { diversity }\end{array}$ & $\begin{array}{c}\text { No. of alleles } \\
\text { per locus }\end{array}$ \\
\hline A & 116 & 1000 & 997 & 107 & $0.605 \pm 0.033$ & $0.028 \pm 0.014$ & $1.110 \pm 0.326$ \\
B & 37 & 1000 & 998 & 114 & $0.719 \pm 0.054$ & $0.032 \pm 0.016$ & $1.122 \pm 0.354$ \\
C & 97 & 1000 & 996 & 105 & $0.395 \pm 0.051$ & $0.021 \pm 0.010$ & $1.108 \pm 0.320$ \\
E & 66 & 1000 & 996 & 100 & $0.406 \pm 0.069$ & $0.021 \pm 0.010$ & $1.101 \pm 0.305$ \\
F & 109 & 1000 & 997 & 83 & $0.595 \pm 0.027$ & $0.029 \pm 0.014$ & $1.085 \pm 0.290$ \\
Wild & 29 & 1000 & 996 & 78 & $0.495 \pm 0.096$ & $0.025 \pm 0.012$ & $1.080 \pm 0.279$ \\
\hline
\end{tabular}

* loci with less than $5 \%$ missing data. 
Table 2. Distribution of the number of haplotypes, based on sequences of the mitochondrial DNA control region in Litopenaeus vannamei broodstock from five hatcheries (A, B, C, E, F) and a wild sample from northwestern Mexico.

Tabla 2. Distribución del número de haplotipos, con base en secuencias de la región control del ADN mitocondrial en el pie de cría de Litopenaeus vannamei de cinco laboratorios (A, B, C, E, F) y de una muestra silvestre del noroeste de México.

\begin{tabular}{|c|c|c|c|c|c|c|}
\hline Haplotype & A & $\mathrm{B}$ & $\mathrm{C}$ & $\mathrm{E}$ & $\mathrm{F}$ & Wild \\
\hline H1 & 39 & 16 & 73 & 10 & 44 & 6 \\
\hline $\mathrm{H} 2$ & 62 & 12 & 20 & 50 & 54 & 20 \\
\hline $\mathrm{H} 3$ & 1 & & & & & \\
\hline $\mathrm{H} 4$ & 1 & & & & & \\
\hline H5 & 1 & & & & & \\
\hline H6 & 2 & & & & & \\
\hline $\mathrm{H} 7$ & 1 & & & & & \\
\hline H8 & 1 & & & & & \\
\hline H9 & 1 & & & & & \\
\hline $\mathrm{H} 10$ & 1 & & & & & \\
\hline H11 & & 1 & & & & \\
\hline H12 & & & & & 1 & \\
\hline H13 & & & & & 1 & \\
\hline H14 & 1 & & & & & \\
\hline H15 & & & & & 1 & \\
\hline H16 & & 1 & & & & \\
\hline H17 & & 1 & & & & \\
\hline H18 & & 1 & & & & \\
\hline H19 & & & 1 & & & \\
\hline H 20 & & & & 1 & & \\
\hline $\mathrm{H} 22$ & & & & & & 1 \\
\hline $\mathrm{H} 23$ & & & & & & 1 \\
\hline $\mathrm{H} 25$ & 2 & & & & 4 & \\
\hline H27 & & & & 3 & 2 & \\
\hline H29 & & & 1 & 2 & & \\
\hline H30 & & & & & & 1 \\
\hline H31 & & & & & 2 & \\
\hline H32 & & 2 & & & & \\
\hline H33 & & 1 & & & & \\
\hline H34 & 1 & & & & & \\
\hline H35 & & 1 & & & & \\
\hline H37 & & 1 & & & & \\
\hline H38 & 1 & & & & & \\
\hline H39 & & & 1 & & & \\
\hline $\mathrm{H} 40$ & 1 & & & & & \\
\hline H41 & & & 1 & & & \\
\hline $\begin{array}{l}\text { No. of } \\
\text { individuals }\end{array}$ & 116 & 37 & 97 & 66 & 109 & 29 \\
\hline $\begin{array}{l}\text { No. of } \\
\text { haplotypes }\end{array}$ & 15 & 10 & 6 & 5 & 9 & 5 \\
\hline
\end{tabular}

La muestra silvestre presentó el menor número de sitios polimórficos (tabla 1).

Dos haplotipos ( $\mathrm{H} 1$ y $\mathrm{H} 2)$ tuvieron una alta frecuencia en todas las muestras, con el 76\% en las muestras del laboratorio B y hasta el $96 \%$ en las del C (tabla 2). Los otros haplotipos tuvieron una frecuencia muy baja y fueron exclusivos de laboratorios individuales, con la excepción del haplotipo 29. Las muestras del laboratorio E y la muestra silvestre presentaron el menor número de haplotipos (5), y las del A presentaron el más alto (15). Las muestras del laboratorio $\mathrm{B}$ presentaron la mayor diversidad y las del $\mathrm{C}$ la menor. La diversidad fue más baja en la muestra silvestre que en las muestras de tres laboratorios (tabla 1).

La diferenciación genética, basada en el AMOVA, demostró que el $86 \%$ de la varianza molecular se da dentro de los laboratorios; el valor global de Fst fue de 0.14 y significativamente diferente de cero $(P<0.001)$. De las 15 comparaciones pareadas, el análisis de Fst mostró que el 66\% fueron significativas, concordando en lo general con la prueba exacta. Ambas pruebas mostraron que la muestra silvestre no fue diferente de la de los laboratorios A, E y F (tabla 3).

Mil ciento nueve sitios nucleotídicos fueron generados en el alineamiento debido a las indels, de los cuales 607 fueron parsimoniosamente informativos, 174 fueron no informativos y 328 resultaron constantes en el análisis. El árbol de máxima parsimonia nucleotídica (longitud $=1683$ pasos, índice de consistencia $=0.708$, índice de retención $=0.880$ ) y el análisis de máxima verosimilitud (longitud $=8669$ pasos, modelo de evolución TVM $+\mathrm{G}$ ) dieron como resultado árboles con una topología muy similar; por tanto, sólo el árbol de máxima verosimilitud se muestra en la figura 1. El árbol filogenético de máxima parsimonia y máxima verosimilitud muestra que las secuencias de los camarones cultivados y la muestra silvestre forman dos grupos (uno para el haplotipo $\mathrm{H} 1$ y otro para el hapoltipo H2), que constituyen un clado bien diferenciado de nuestras secuencias, sugiriendo un sólo ancestro común para todos los reproductores utilizados en el noroeste de México. Las otras secuencias de L. vannamei silvestre del GenBank (AY845710-AY845715) forman un clado hermano del grupo de reproductores estudiados, y todos resultan de un sólo ancestro común de camarón blanco y muestran un fuerte soporte estadístico (100\%) para la condición monofilética. Además, el clado que engloba a todos los peneidos muestra una estrecha relación entre las especies de la familia (fig. 1).

\section{DISCUSIÓN}

De acuerdo con los camaronicultores y los responsables de los laboratorios de producción de postlarvas, el origen de los actuales linajes de camarón en México es de un lote importado de Venezuela en 1997 que sobrevivió a una mortalidad por síndrome de Taura (Perez-Enriquez et al. 2009). El análisis realizado por Perez-Enriquez et al. (2009) basado en 
Table 3. Genetic differentiation among the samples from the shrimp hatcheries (A, B, C, E, F) and the wild sample. The $F_{S T}$ values and their significance are given above the diagonal, and the $P$ values of the exact test are given below the diagonal.

Tabla 3. Diferenciación genética entre los laboratorios productores de larvas de camarón (A, B, C, E, F) y la muestra silvestre. Arriba de la diagonal se indican los valores de $F_{S T}$ y su significancia, y debajo de la diagonal se indican los valores de significancia de la prueba exacta.

\begin{tabular}{lcccccc}
\hline Sample & $\mathrm{A}$ & $\mathrm{B}$ & $\mathrm{C}$ & $\mathrm{E}$ & $\mathrm{F}$ & Wild \\
\hline $\mathrm{A}$ & & $0.043^{*}$ & $0.188^{* * *}$ & $0.090^{* * *}$ & $-0.005 \mathrm{~ns}$ & $0.026 \mathrm{~ns}$ \\
$\mathrm{~B}$ & 0.011 & & $0.055^{*}$ & $0.248^{* * *}$ & $0.023 \mathrm{~ns}$ & $0.145^{* * *}$ \\
$\mathrm{C}$ & 0.000 & 0.000 & & $0.443^{* * *}$ & $0.158^{* * *}$ & $0.360^{* * *}$ \\
E & 0.003 & 0.000 & 0.000 & & $0.114^{* * *}$ & $-0.005 \mathrm{~ns}$ \\
F & 0.227 & 0.004 & 0.000 & 0.000 & $0.069 \mathrm{~ns}$ \\
Wild & 0.446 & 0.013 & 0.000 & 0.176 & 0.045 \\
\hline
\end{tabular}

$*=0.01<\mathrm{P}<0.05 ; * * *=\mathrm{P}<0.001 ; \mathrm{ns}=$ not significant.

174 were not informative, and 328 were constant in the analysis. The nucleotide maximum parsimony tree (length $=1683$ steps, consistence index $=0.708$, retention index $=0.880$ ) and maximum likelihood analysis (length $=8669$ steps, evolution model TVM + G) produced trees with highly similar topology. Thus, only the maximum likelihood tree is shown in figure 1. The phylogenetic tree of maximum parsimony and maximum likelihood shows that sequences of cultivated shrimp and the wild shrimp form two groups (one for haplotype H1 and another for haplotype H2), which constitute a distinct clade of our sequences, suggesting a single common ancestor for all broodstock used in northwestern Mexico. The other GenBank sequences of wild L. vannamei (AY845710-AY845715) form a sister clade of the studied group of broodstock, and all result from a single common ancestor and show strong statistical support (100\%) for the monophyletic condition. In addition, the clade that includes all penaeids shows a close relationship between the species of the family (fig. 1).

\section{DISCUSSION}

According to shrimp growers and hatchery managers, the origin of current shrimp strains in Mexico is a batch imported from Venezuela in 1997 that survived a Taura syndrome outbreak (Perez-Enriquez et al. 2009). The analysis carried out by Perez-Enriquez et al. (2009) based on microsatellite allelic composition indicated the recent common origin of the Mexican broodstock. Our study agrees and strengthens the hypothesis of a common ancestor, based on the abundance of two genetically distant haplotypes present in shrimp from all five hatcheries and the high percentage of paired comparisons with no significant differences ( $F_{S T}$ and exact test). The two haplotypes may have increased their frequencies by domestication and selection in Venezuela or even in the founding group in Texas (Sunden and Davis 1991, PerezEnriquez et al. 2009). The genetic structure of cultivated shrimp and differentiation from wild populations is depicted in the phylogenetic tree, where none of the previously reported sequences in GenBank of wild whiteleg shrimp were included in the cultivated shrimp clade. la composición alélica microsatelital sugirió un origen común reciente del pie de cría mexicano. Nuestro estudio concuerda y fortalece la hipótesis de un ancestro común debido a la abundancia de dos haplotipos genéticamente distantes y presentes en los cinco laboratorios analizados, y al alto porcentaje de comparaciones pareadas sin diferencias significativas (Fst y prueba exacta). Los dos haplotipos pudieron haber incrementado su frecuencia por el proceso de domesticación selectiva en Venezuela o incluso en el grupo fundador en Texas (Sunden y Davis 1991, Perez-Enriquez et al. 2009). La estructura genética de los camarones cultivados y la diferenciación de las poblaciones silvestres se muestra en el árbol filogenético, en donde ninguna de las secuencias de camarones silvestres previamente registradas en el GenBank se incluyeron en el clado de los camarones cultivados.

En el cultivo de camarón, como en los programas de animales de granja, la cruza de individuos con lazos de parentesco o el mal manejo del pie de cría generalmente da lugar a la endogamia y a la pérdida de variabilidad genética. Los efectos nocivos de la endogamia en organismos domesticados se han documentado ampliamente (Goyard et al. 2003, De Donato et al. 2005, Moss et al. 2007). El alto valor de endogamia es bien conocido en el camarón cultivado en el noroeste de México con respecto a las poblaciones silvestres (Ramos-Paredes y Grijalva-Chon 2003, Soto-Hernández y Grijalva-Chon 2004, Perez-Enriquez et al. 2009). PerezEnriquez et al. (2009) registraron altos valores de coeficiente de endogamia en los mismos laboratorios que se estudiaron en el presente estudio. Sus resultados concuerdan con los nuestros con respecto a la baja variabilidad genética, la alta identidad genética y la baja diferenciación entre los laboratorios. Aunque la endogamia no pueda ser estimada utilizando marcadores mitocondriales, éstos sí pueden dar una información adecuada de la diversidad genética. La baja diversidad del pie de cría analizado en el presente estudio, en comparación con la diversidad registrada por Valles-Jiménez et al. (2006) para poblaciones silvestres, y la evidente alta frecuencia de dos haplotipos indican que el camarón cultivado de los laboratorios analizados muestran una combinación de efecto fundador, deriva genética y selección. 


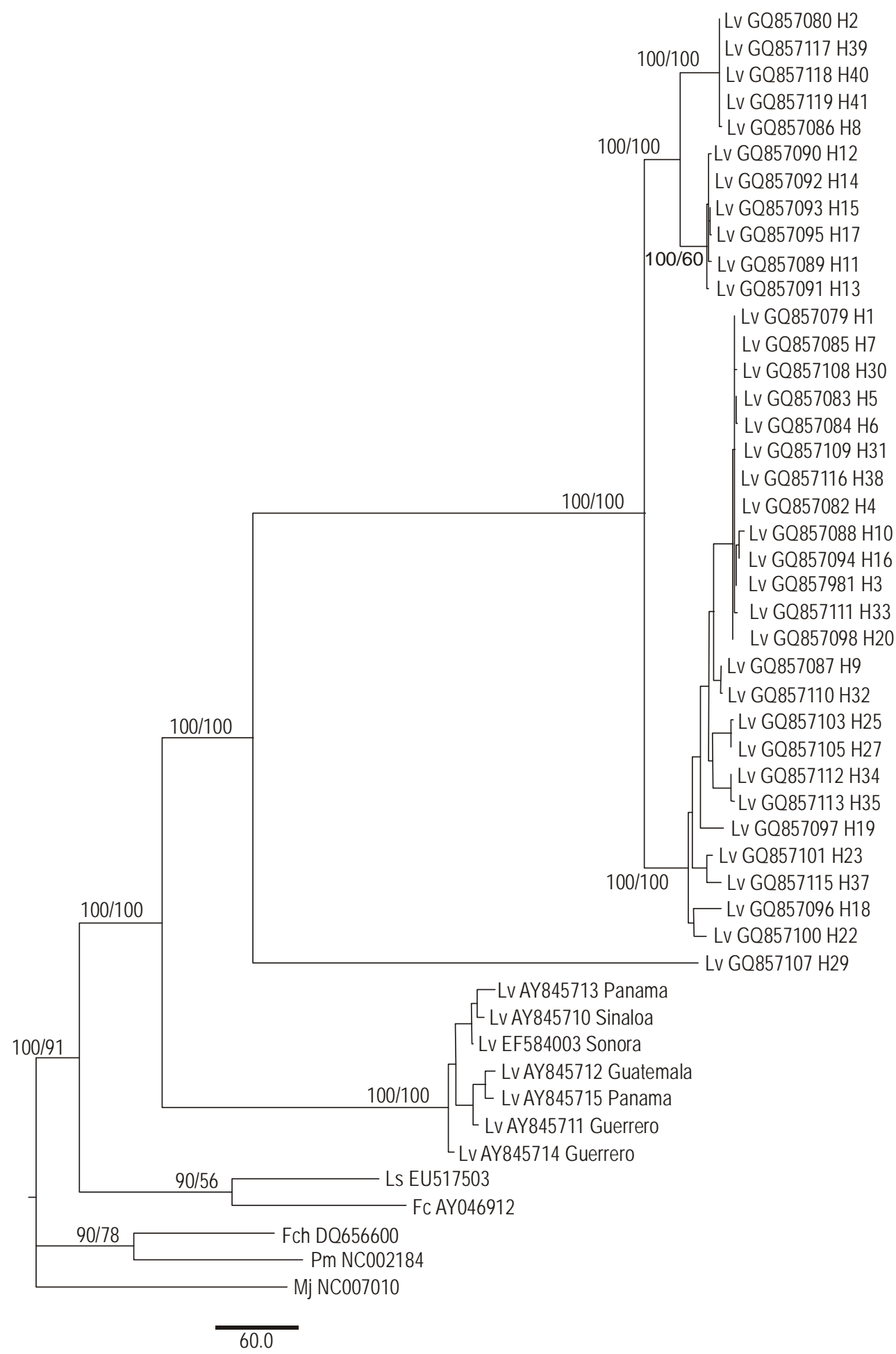

Figure 1. Phylogenetic tree derived for the mitochondrial DNA control region of Litopenaeus vannamei by maximum parsimony and maximum likelihood analysis in PAUP* and based on nucleotide sequences of penaeid shrimp. Numbers at the nodes are the bootstrap percentages for 1000 replicates for maximum parsimony/maximum likelihood. GenBank accession numbers for the sequences are indicated. The haplotypes of the samples are those indicated as H1 through $\mathrm{H} 41$.

Figura 1. Árbol filogenético obtenido de la región control del ADN mitocondrial de Litopenaeus vannamei por medio del análisis de máxima parsimonia y máxima verosimilitud con el programa PAUP*. Los números en los nodos son los porcentajes de remuestreo para 1000 réplicas de máxima parsimonia/máxima verosimilitud. Se indican los números de acceso para las secuencias del GenBank. Los haplotipos de las muestras son los indicados como $\mathrm{H} 1$ hasta $\mathrm{H} 41$.

$\mathrm{Lv}=$ Litopenaeus vannamei, $\mathrm{Ls}=$ Litopenaeus stylirostris, $\mathrm{Fc}=$ Farfantepenaeus californiensis, Fch = Fenneropenaeus chinensis, $\mathrm{Pm}=$ Penaeus monodon, $\mathrm{Mj}=$ Marsupenaeus japonicus . 
In shrimp cultivation, as in livestock programs, breeding individuals that are related or the mismanagement of broodstock usually causes inbreeding and loss of genetic variability. The detrimental effects of inbreeding on domesticated animals have been widely reported (Goyard et al. 2003, De Donato et al. 2005, Moss et al. 2007). For cultivated shrimp, high inbreeding with respect to wild populations in northwestern Mexico is well known (Ramos-Paredes and GrijalvaChon 2003, Soto-Hernández and Grijalva-Chon 2004, PerezEnriquez et al. 2009). Perez-Enriquez et al. (2009) report high inbreeding coefficients at the same hatcheries involved in our study. Their results agree with our findings: low genetic variability, high genetic identity, and low genetic differentiation among the hatcheries. Even though inbreeding cannot be estimated using mtDNA markers, they provide information about genetic diversity. The low diversity of broodstock analyzed in our study, compared with the diversity analyzed by Valles-Jiménez et al. (2006) for wild populations, and the high frequency of two haplotypes indicate that cultivated shrimp from these hatcheries show a combination of founder effect, genetic drift, and selection.

Despite the common origin of the sample lots, diversity varies among hatcheries. Significant differences in $F_{S T}$ values were found between some paired tests, which may be the result of genetic drift; however, since all samples have the same two very frequent haplotypes, genetic drift would have occurred in a very similar way in all of them, and that can only happen if the original genetic composition of each hatchery was very similar. If the genetic composition had been different, the process of genetic drift would have generated a different haplotype composition among hatcheries. Furthermore, the diversity pattern among hatcheries (those with the maximum and minimum levels) does not coincide with the pattern reported by Perez-Enriquez et al. (2009), possibly because there are differences in the mtDNA and microsatellite evolution rates. The population bottleneck after the outbreak of the Taura syndrome virus in the late 1990s (Perez-Enriquez et al. 2009) could have generated a different population genetic structure in mitochondrial and nuclear genomes because the mitochondrial genome is more prone to be structured than the nuclear genome (Ferris and Berg 1987, Birky et al. 1989). The Hardy-Weinberg disequilibrium could be caused by that bottleneck (Perez-Enriquez et al. 2009). Additionally, the phylogenetic analysis that depicted the cultivated shrimp in a separate clade supports the bottleneck hypothesis.

Contrary to reports on wild populations (Klinbunga et al. 1998, 1999, 2001; McMillen-Jackson and Bert 2003; VallesJiménez et al. 2006; Khamnamtong et al. 2009), the wild sample in our study had less diversity than shrimp from some of the hatcheries. This suggests a closer relationship with cultivated shrimp because shrimp escape from farms. Several genetic tools have been used to assess the population genetic structure and diversity of wild and cultivated populations of penaeid shrimp (table 4). The diversity values obtained in our
A pesar del origen común de los lotes ofertados por los laboratorios, la diversidad no es la misma entre ellos. Se encontraron diferencias significativas en los valores de Fst entre algunas pruebas pareadas, lo cual puede ser el resultado de la deriva genética. Sin embargo, el hecho de que todas las muestras tengan los mismos dos haplotipos con alta frecuencia indica que el proceso de deriva genética ocurrió de una manera muy similar en todos ellos, y eso sólo puede ocurrir si la composición genética original de cada laboratorio fue muy semejante. Si la composición genética hubiese sido distinta, el proceso de deriva posterior hubiera generado una composición haplotípica diferente entre los laboratorios. Además, el patrón de diversidad entre los laboratorios (con los niveles máximos y mínimos) no coincide con el patrón registrado por Perez-Enriquez et al. (2009), posiblemente debido a las diferentes tasas de evolución del ADN mitocondrial y los microsatélites. El cuello de botella poblacional después de la epizootia causada por el virus del síndrome de Taura al final de la década de los noventa (Perez-Enriquez et al. 2009) pudo haber generado una estructura genética poblacional diferente en los genomas mitocondrial y nuclear, ya que el genoma mitocondrial es más propenso a estructurarse que el genoma nuclear (Ferris y Berg 1987, Birky et al. 1989). El desequilibro de Hardy-Weinberg pudo haber sido causado por ese cuello de botella (Perez-Enriquez et al. 2009). Adicionalmente, el resultado del análisis filogenético que muestra a los camarones cultivados en un clado independiente del de los camarones registrados en el GenBank sostiene ésta hipótesis de cuello de botella.

Contrario a los reportes sobre poblaciones silvestres (Klinbunga et al. 1998, 1999, 2001; McMillen-Jackson y Bert 2003, Valles-Jiménez et al. 2006, Khamnamtong et al. 2009), la muestra silvestre analizada en este estudio presentó una diversidad menor que la de los organismos de algunos de los laboratorios. Esto sugiere una estrecha relación con los camarones cultivados debido a que los organismos se escapan de las granjas. Se han utilizado varias herramientas genéticas para evaluar la estructura genética poblacional y la diversidad de poblaciones silvestres y cultivadas de camarones peneidos (tabla 4). Los valores de diversidad obtenidos en este estudio no son comparables con los estudios que utilizan otras herramientas de análisis, tal y como se evidenció al comparar nuestros resultados con los de Perez-Enriquez et al. (2009). La carencia de estudios poblacionales que contemplen la secuenciación completa de la región control mitocondrial de L. vannamei o de otros peneidos hace difícil evaluar los valores de diversidad genética de la especie. McMillenJackson y Bert (2003) obtuvieron datos de diversidad de nucleótidos para poblaciones silvestres de Farfantepenaeus aztecus y Litopenaeus setiferus, pero de sólo una fracción de la región control mitocondrial (541 y 499 pb, respectivamente), y obtuvieron valores promedio de $2.8 \%$ y $2.1 \%$, respectivamente, los cuales son cercanos al promedio de $2.6 \%$ obtenido en este estudio. Sin embargo, Kumar et al. 
Table 4. Genetic tools used in studies of wild and cultivated penaeid shrimp. COI, citochrome oxidase I; 16S rRNA, ribosomal RNA 16S gene; RFLP, restriction fragment length polymorphism; RAPD, random amplified polymorphic DNA; VNTR, variable number of tandem repeats; and PCR SSCP EST, polymerase chain reaction single strand conformation polymorphism of expressed sequence tags.

Tabla 4. Herramientas genéticas de análisis utilizadas en camarones peneidos silvestres y cultivados. COI, citocromo oxidasa I;

16S rRNA, gen del ARN ribosomal 16S; RFLP, polimorfismo en la longitud de los fragmentos de restricción; RAPD, ADN polimórfico amplificado al azar; VNTR, número variable de repeticiones en paquete; y PCR SSCP EST, polimorfismo de conformación de simple banda de marcadores de secuencias expresadas y amplificadas por la reacción en cadena de la polimerasa.

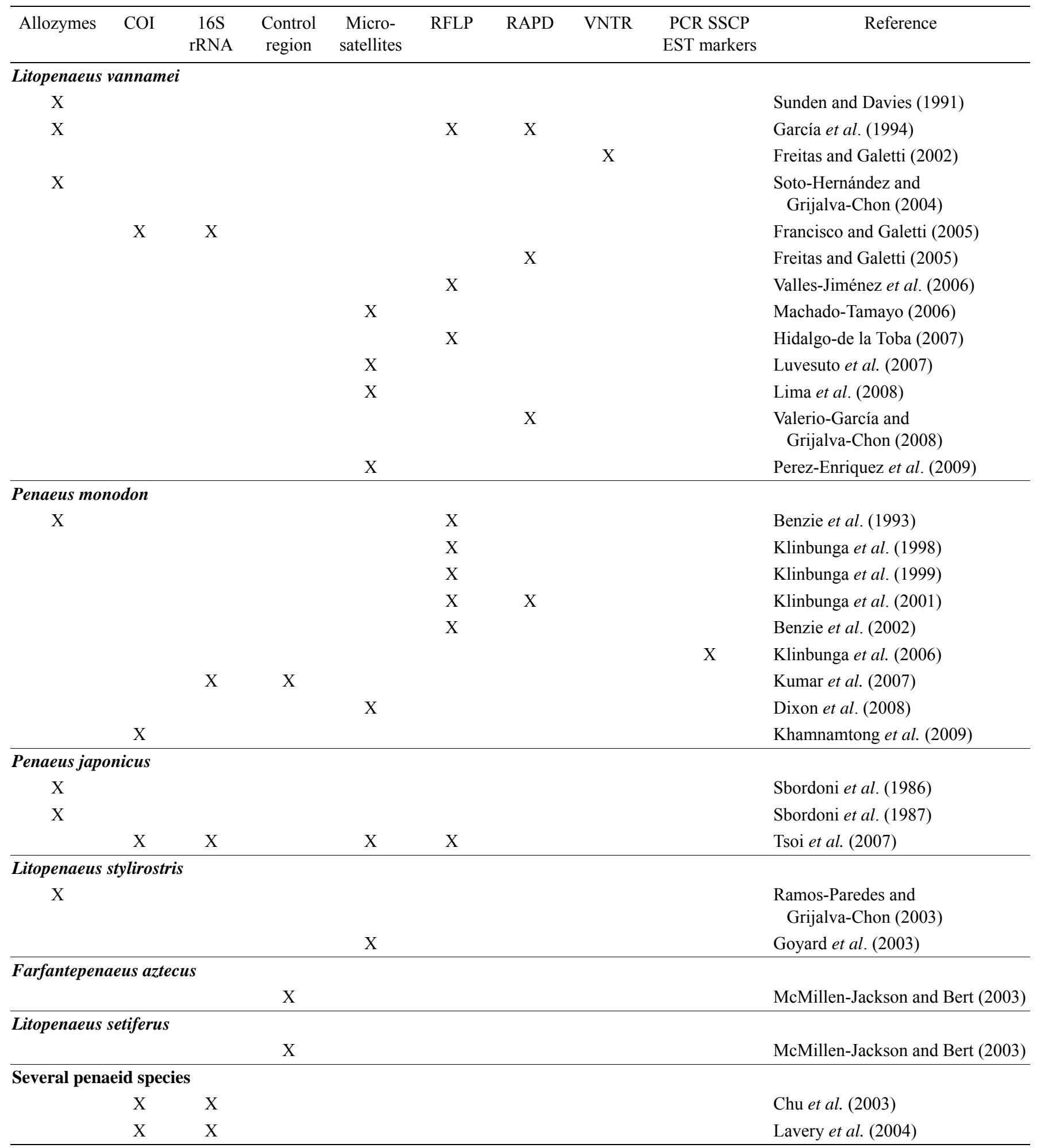


study are not comparable with studies using other analytical tools, as seen by comparing our results with those of PerezEnriquez et al. (2009). The lack of population studies that consider the complete sequencing of the mitochondrial control region of $L$. vannamei and other penaeids makes it difficult to assess genetic diversity values of whiteleg shrimp. McMillen-Jackson and Bert (2003) obtained data on nucleotide diversity from wild populations of Farfantepenaeus aztecus and Litopenaeus setiferus, but only from a fraction of the mitochondrial control region (541 and $499 \mathrm{bp}$, respectively), resulting in average values of $2.8 \%$ and $2.1 \%$, respectively, which are close to the average values of $2.6 \%$ obtained in our study. Kumar et al. (2007) reported an average of 3.4\% for a fraction of $577 \mathrm{bp}$ of the mitochondrial control region of wild Penaeus monodon, with values as high as 5.4\% for one of the populations in India. The values reported by Kumar et al. (2007) are high, considering that we analyzed the entire control region and the maximum value obtained was $3.2 \%$. Since the whiteleg shrimp come from a long, artificial selection process, this inevitably leads to declining diversity.

In spite of our estimates of reduced genetic diversity and the high inbreeding reported by Soto-Hernández and Grijalva-Chon (2004) and Perez-Enriquez et al. (2009), there is no evidence of inbreeding depression in shrimp cultivated in Mexico, given the sustained growth of the industry, from 45,000 $\mathrm{t}$ in 2000 to $133,282 \mathrm{t}$ in 2009 (CONAPESCA 2010, 2011). However, this dynamic growth has declined in recent years $(109,815 \mathrm{t}$ in 2011 and $100,320 \mathrm{t}$ in 2012), caused mainly by viral outbreaks (white spot syndrome), particularly in the state of Sonora (Aquaculture Health Committee of the State of Sonora, pers. comm.; CONAPESCA 2012). Among the farming practices that need to be improved is the management of genetic diversity in hatcheries. The recommendation for this is to enrich the gene pool of broodstock through the exchange of pathogen-free shrimp from hatcheries that maintain lineages other than those reported herein, at a rate that does not affect overall performance and following strict reproduction control to avoid inbreeding in future generations.

\section{ACKNOWLEDGMENTS}

This research was funded by Consejo Nacional de Ciencia y Tecnología (CONACYT, Mexico, research project 2006-60030 to RPE). FMC was a recipient of a graduate fellowship from CONACYT (No. 208901). Thanks are due to the commercial hatcheries that provided the samples, and to Jorge Hernández-López, Daniel Coronado-Molina, Trinidad Encinas-García, and Arturo Sánchez-Paz at CIBNOR (Hermosillo) for technical support. Ira Fogel (CIBNOR, La Paz) provided English editing services.
(2007) registraron un promedio de 3.4\% para una fracción de 577 pb de la región control mitocondrial de Penaeus monodon silvestre, con valores tan altos como el 5.4\% registrado para una de las poblaciones analizadas en la India. Los valores registrados por Kumar et al. (2007) son elevados, considerando que nosotros analizamos la totalidad de la región control y el valor máximo obtenido fue de $3.2 \%$. Se debe considerar que los organismos de L. vannamei analizados provienen de un largo proceso de selección artificial, lo cual conduce forzosamente a una disminución de la diversidad.

A pesar de la reducida diversidad genética estimada en los lotes analizados en el presente estudio y la alta endogamia registrada por Soto-Hernandez y Grijalva-Chon (2004) y Perez-Enriquez et al. (2009), no hay evidencia de una depresión endogámica en el camarón cultivado en México ya que se ha mantenido el crecimiento sostenido (promedio del 18\%) de la actividad, de 45,000 t en 2000 a 133,282 t en 2009 (CONAPESCA 2010, 2011). Sin embargo, este crecimiento activo se ha visto reducido en años recientes $(109,815 \mathrm{t}$ en 2011 y 100,320 t en 2012) debido principalmente a mortalidades de origen viral, particularmente en el estado de Sonora (Comité de Sanidad Acuícola del Estado de Sonora, com. pers.; CONAPESCA 2012). Esto obliga a mejorar las prácticas de cultivo, entre las cuales el manejo de la diversidad genética en las unidades de producción de postlarvas es una de las más importantes. La recomendación para ello es el enriquecimiento del acervo genético de los lotes de reproductores a través del intercambio de organismos libres de patógenos provenientes de laboratorios que mantengan linajes distintos a los aquí registrados, a una tasa que no afecte el desempeño alcanzado y siguiendo un estricto control de la reproducción a fin de evitar la acumulación de la endogamia en las generaciones futuras.

\section{Agradecimientos}

Esta investigación fue financiada por el Consejo Nacional de Ciencia y Tecnología (CONACYT, México) a través del proyecto nnúmero 2006-60030 otorgado a RPE. El CONACYT otorgó una beca de posgrado (No. 208901) a FMC. Agradecemos a los laboratorios de producción que contribuyeron con las muestras, y a Jorge Hernández-López, Daniel Coronado-Molina, Trinidad Encinas-García y Arturo Sánchez-Paz del CIBNOR (Hermosillo) el apoyo técnico. Ira Fogel del CIBNOR (La Paz) contribuyó con la edición de la versión en inglés. 


\section{REFERENCES}

Álvarez-Jurado A. 1987. Genética y acuacultura. In: Espinosa J, Labarta U (eds.), Genética en Acuacultura. Industrias Gráficas España, Madrid, pp. 1-31.

Benzie JAH. 2009. Use and exchange of genetic resources of penaeid shrimps for food and aquaculture. Rev. Aquaculture 1: $232-250$. http://dx.doi.org/10.1111/j.1753-5131.2009.01018.x

Benzie JAH, Williams ST. 1996. Limitations in the genetic variation of hatchery produced batches of the giant clam, Tridacna gigas. Aquaculture 139: 225-241. http://dx.doi.org/10.1016/0044-8486(95)01171-4

Benzie JAH, Ballment E, Frusher S. 1993. Genetic structure of Penaeus monodon in Australia: Concordant results from mtDNA and allozymes. Aquaculture 111: 89-93. http://dx.doi.org/10.1016/0044-8486(93)90027-V

Benzie JAH, Ballment E, Forbes AT, Demetriades NT, Sugama K, Haryanti, Moria S. 2002. Mitochondrial DNA variation in IndoPacific populations of the giant tiger prawn, Penaeus monodon. Mol. Ecol. 11: 2553-2559.

http://dx.doi.org/10.1046/j.1365-294X.2002.01638.x

Birky CW Jr, Fuerst P, Maruyama T. 1989. Organelle gene diversity under migration, mutation, and drift: Equilibrium expectations, approach to equilibrium, effects of heteroplasmic cells, and comparison to nuclear genes. Genetics 121: 613-627.

Bremer K. 1994. Branch support and tree stability. Cladistics 10: 295-304. http://dx.doi.org/10.1006/clad.1994.1019

Chu KH, Li CP, Tam YK, Lavery S. 2003. Application of mitochondrial control region in population genetic studies of the shrimp Penaeus. Mol. Ecol. Notes 3: 120-122. http://dx.doi.org/10.1046/j.1471-8286.2003.00376.x

CONAPESCA 2010. Anuario Estadístico de Acuacultura y Pesca. Secretaría de Agricultura, Ganadería, Desarrollo Rural, Pesca y Alimentación. Comisión Nacional de Pesca y Acuacultura, Mexico City, http://www.conapesca.sagarpa.gob.mx/wb/cona/ cona_anuario_estadistico_de_pesca.

CONAPESCA 2011. Anuario Estadístico de Acuacultura y Pesca. Secretaría de Agricultura, Ganadería, Desarrollo Rural, Pesca y Alimentación. Comisión Nacional de Pesca y Acuacultura, Mexico City, http://www.conapesca.sagarpa.gob.mx/wb/cona/ cona_anuario_estadistico_de pesca.

CONAPESCA 2012. Anuario Estadístico de Acuacultura y Pesca. Secretaría de Agricultura, Ganadería, Desarrollo Rural, Pesca y Alimentación. Comisión Nacional de Pesca y Acuacultura, Mexico City, http://www.conapesca.sagarpa.gob.mx/wb/cona/ cona_anuario_estadistico_de_pesca.

De Donato M, Manrique R, Ramirez R, Mayer L, Howell C. 2005. Mass selection and inbreeding effects on a cultivated strain of Penaeus (Litopenaeus) vannamei in Venezuela. Aquaculture 247: $159-167$. http://dx.doi.org/10.1016/j.aquaculture.2005.02.005

Dixon TJ, Coman GJ, Arnold SJ, Sellars MJ, Lyons RE, Dierens L, Preston NP, Li Y. 2008. Shifts in genetic diversity during domestication of black tiger shrimp, Penaeus monodon, monitored using two multiplexed microsatellite systems. Aquaculture 283: 1-6. http://dx.doi.org/10.1016/j.aquaculture.2008.07.009

Excoffier L, Laval G, Schneider S. 2005. Arlequin ver. 3.0: An integrated software package for population genetics data analysis. Evol. Bioinformatics 1: 47-50.

Felsenstein J. 1985. Confidence limits on phylogenies: An approach using the bootstrap. Evolution 39: 783-791. http://dx.doi.org/10.2307/2408678
Ferris SD, Berg WJ. 1987. The utility of mitochondrial DNA in fish genetics and fishery management. In: Ryman N, Utter F (eds.), Population Genetics and Fishery Management. University of Washington Press, Seattle, pp. 277-299.

Francisco AK, Galetti PM Jr. 2005. Genetic distance between broodstocks of the marine shrimp Litopenaeus vannamei (Decapoda, Penaeidae) by mtDNA analysis. Genet. Mol. Biol. 28: 258-261. http://dx.doi.org/10.1590/S1415-47572005000200014

Freitas PD, Galetti PM Jr. 2002. PCR-based VNTR core sequence analysis for inferring genetic diversity in the shrimp Litopenaeus vannamei. Genet. Mol. Biol. 4: 431-434. http://dx.doi.org/10.1590/S1415-47572002000400012

Freitas P, Galetti PM Jr. 2005. Assessment of the genetic diversity in five generations of a commercial broodstock line of Litopenaeus vannamei shrimp. Afr. J. Biotechnol. 12: 1362-1367.

García DK, Faggart MA, Rhoades L, Alcivar-Warren A. 1994. Genetic diversity of cultured Penaeus vannamei shrimp using three molecular genetic techniques. Mol. Mar. Biol. Res. 5: 270-280.

Goyard E, Arnaud S, Vonau V, Bishoff V, Mouchel O, Pham D. 2003. Residual genetic variability in domesticated populations of the Pacific blue shrimp (Litopenaeus stylirostris) of New Caledonia, French Polynesia and Hawaii and some management recommendations. Aquat. Living Resour. 16: 501-508. http://dx.doi.org/10.1016/j.aquliv.2003.07.001

Hidalgo-de la Toba JA. 2007. Evaluación de la diversidad genética en el camarón blanco (Litopenaeus vannamei) en granjas de cultivo de Nayarit, México, mediante análisis de RFLP de la región control del ADNmt. BSc thesis, Universidad Autónoma de Baja California Sur, La Paz, BCS, México. 60 pp.

Khamnamtong B, Klinbunga S, Menasveta P. 2009. Genetic diversity and geographic differentiation of the giant tiger shrimp (Penaeus monodon) in Thailand analyzed by mitochondrial COI sequences. Biochem. Genet. 47: 42-55. http://dx.doi.org/10.1007/s10528-008-9205-3

Klinbunga S, Penman DJ, McAndrew BJ. 1998. A preliminary study of ribosomal DNA polymorphism in the tiger shrimp Penaeus monodon. J. Mar. Biotechnol. 6: 186-188.

Klinbunga S, Penman DJ, McAndrew BJ, Tassanakajon A. 1999. Mitochondrial DNA diversity in three populations of the giant tiger shrimp Penaeus monodon. Mar. Biotechnol. 1: 113-121. http://dx.doi.org/10.1007/PL00011758

Klinbunga S, Siludjai D, Wudthijinda W, Tassanakajon A, Jarayabhand P, Menasveta P. 2001. Genetic heterogeneity of the giant tiger shrimp (Penaeus monodon) in Thailand revealed by RAPD and mitochondrial DNA RFLP analyses. Mar. Biotechnol. 3: 428-438. http://dx.doi.org/10.1007/s10126-001-0055-9

Klinbunga S, Preechaphol R, Thumrungtanakit S, Leelatanawit R, Aoki T, Jarayabhand P, Menasveta P. 2006. Genetic diversity of the giant tiger shrimp (Penaeus monodon) in Thailand revealed by PCR-SSCP of polymorphic EST-derived markers. Biochem. Genet. 44: 222-236. http://dx.doi.org/ 10.1007/s10528-006-9027-0

Kumar N, Lakra WS, Majumdar KC, Goswami M, Kondadhasula R. 2007. Genetic diversity in the Indian population of Penaeus monodon (Fabricius 1798) as revealed by mtDNA sequence analysis. Aquacult. Res. 38: 862-869. http://dx.doi.org/10.1111/j.1365-2109.2007.01740.x

Larkin MA, Blackshields G, Brown NP, Chenna R, McGettigan PA, McWilliam H, Valentin F, Wallace IM, Wilm A, Lopez R, Thompson JD, Gibson TJ, Higgins DG. 2007. Clustal W and Clustal X version 2.0. Bioinformatics 23: 2947-2948. http://dx.doi.org/10.1093/bioinformatics/btm404 
Lavery S, Chan TY, Tam YK, Chu KH. 2004. Phylogenetic relationships and evolutionary history of the shrimp genus Penaeus s.I. derived from mitochondrial DNA. Mol. Phylogenet. Evol. 31: 39-49. http://dx.doi.org/10.1016/j.ympev.2003.07.015

Lima APS, Lira dos Santos AC, Dantas HL, Gomes-Filho MA, Maggioni R, Moura-Coimbra MR. 2008. Genetic monitoring of broodstock of the marine shrimp Litopenaeus vannamei in a closed rearing system in Pernambuco, Brazil. Aquacult. Res. 39: 1461-1466. http://dx.doi.org/10.1111/j.1365-2109.2008.02022.x

Luvesuto E, Freitas PD, Galetti Jr PM. 2007. Genetic variation in a closed line of the white shrimp Litopenaeus vannamei (Penaeidae). Genet. Mol. Biol. 30: 1156-1160. http://dx.doi.org/10.1590/S1415-47572007000600021

Machado-Tamayo RJ. 2006. Assessment of genetic variability in two lots of white shrimp, Litopenaeus vannamei (Boone 1931) introduced to Cuba. MSc thesis, Norwegian College of Fishery Science, University of Tromsø, Norway, 42 pp.

McMillen-Jackson AL, Bert TM. 2003. Disparate patterns of population genetic structure and population history in two sympatric penaeid shrimp species (Farfantepenaeus aztecus and Litopenaeus setiferus) in the eastern United States. Mol. Ecol. 12: 2895-2905. http://dx.doi.org/10.1046/j.1365-294X.2003.01955.x

Moss DR, Arce SM, Otoshi CA, Doyle RW, Moss SM. 2007. Effects of inbreeding on survival and growth of Pacific white shrimp Penaeus (Litopenaeus) vannamei. Aquaculture 272: 30-37. http://dx.doi.org/10.1016/j.aquaculture.2007.08.014

Perez-Enriquez R, Hernández-Martínez F, Cruz P. 2009. Genetic diversity status of white shrimp Penaeus (Litopenaeus) vannamei broodstock in Mexico. Aquaculture 297: 44-50. http://dx.doi.org/10.1016/j.aquaculture.2009.08.038

Posada D, Buckley TR. 2004. Model selection and model averaging in phylogenetics: Advantages of the AIC and Bayesian approaches over likelihood ratio tests. Syst. Biol. 53: 793-808. http://dx.doi.org/10.1080/10635150490522304

Posada D, Crandall KA. 1998. Modeltest: Testing the model of DNA substitution. Bioinformatics 14: 817-818. http://dx.doi.org/10.1093/bioinformatics/14.9.817

Ramos-Paredes J, Grijalva-Chon JM. 2003. Allozyme genetic analysis in hatchery strains and wild blue shrimp, Penaeus (Litopenaeus) stylirostris (Stimpson), from the Gulf of
California. Aquacult. Res. 34: 221-234.

http://dx.doi.org/10.1046/j.1365-2109.2003.00807.x

Rivera-García M, Grijalva-Chon JM. 2006. Genetic variability and differentiation in cultured white shrimp Penaeus (Litopenaeus) vannamei with low and high growth. Cienc. Mar. 32: 1-11.

Sbordoni V, de Matthaeis E, Cobolli-Sbordoni M, La Rosa G, Mattocia M. 1986. Bottleneck effects and the depression of genetic variability in hatchery stocks of Penaeus japonicus (Crustacea, Decapoda). Aquaculture 57: 239-251. http://dx.doi.org/10.1016/0044-8486(86)90202-4

Sbordoni V, La Rosa G, Mattocia M, Cobolli-Sbordoni M, de Matthaeis E. 1987. Genetic changes in seven hatchery stocks of the kuruma prawn, Penaeus japonicus (Crustacea, Decapoda). Proc. World Symp. on Selection, Hybridization and Genetic Engineering in Aquaculture, Bordeaux, 27-30 May 1986.

Soto-Hernández J, Grijalva-Chon JM. 2004. Genetic differentiation in hatchery strains and wild white shrimp Penaeus (Litopenaeus) vannamei (Boone 1931) from northwest Mexico. Aquacult. Int. 12: 593-601. http://dx.doi.org/10.1007/s10499-004-5750-x

Sunden SLF, Davis SK. 1991. Evaluation of genetic variation in a domestic population of Penaeus vannamei (Boone): A comparison with three natural populations. Aquaculture 97: 131-142. http://dx.doi.org/10.1016/0044-8486(91)90260-E

Swofford DL. 2002. PAUP* 4.0: Phylogenetic Analysis Using Parsimony (and other methods) 4.0 Beta. Sinauer Associates, Sunderland, Massachussets, http://www.sinauer.com/paupphylogenetic-analysis-using-parsimony-and-other-methods-4-0beta.html.

Tsoi KH, Chan TY, Chu KH. 2007. Molecular population structure of kuruma shrimp Penaeus japonicus species complex in western Pacific. Mar. Biol. 150: 1345-1364 http://dx.doi.org/10.1007/s00227-006-0426-x

Valerio-García RC, Grijalva-Chon JM. 2008. Random-amplified polymorphic DNA analysis in hatchery populations and wild Pacific white shrimp Penaeus vannamei from the Gulf of California. Aquacult. Res. 39: 666-669. http://dx.doi.org/10.1111/j.1365-2109.2008.01909.x

Valles-Jiménez R, Gaffney PM, Perez-Enriquez R. 2006. RFLP analysis of the mtDNA control region in white shrimp (Litopenaeus vannamei) populations from the eastern Pacific. Mar. Biol. 148: 867-873. http://dx.doi.org/10.1007/s00227-005-0122-2

Received January 2013, received in revised form June 2013, accepted July 2013. 